\title{
Flickering in Cataclysmic Variables: Inventory and Perspectives
}

\author{
Albert Bruch \\ Astronomisches Institut, Wilhelm-Klemm-Str. 10, 48149 Münster, F.R.G.
}

\begin{abstract}
Stochastic variations on short time scales called flickering are a characteristic feature in the light curves of CVs. Their amplitudes indicate that their origin must be related to one of the principle light sources of these systems. But the physical mechanism behind the flickering is unknown. New attempts to quantify the properties of flickering are presented, and a consistent scenario for its origin is outlined.
\end{abstract}

\section{Phenomenology of flickering}

Cataclysmic variables (CVs) are interactive binary stars where a white dwarf primary receives matter via an accretion disk from a late type secondary star. In most systems the optical and ultraviolet light is dominated by the accretion disk. But a considerable fraction of the total radiation is emitted by a unsteady light source which so far has not been identified with certainty and which is responsible for the phenomenon of flickering in CVs.

Any light curve of a $\mathrm{CV}$ in any photometric state (except for classical and recurrent novae in outburst) with a time resolution of the order of seconds reveals more or less strong irregular variations, appearing as a continuous series of flares with a duration of seconds to minutes, depending on their amplitudes. These variations are called flickering. As an example, Fig. 1 shows an unfiltered light curve of the novalike variable TT Ari.

While the total amplitude of the flickering is approximately constant for a given system in a given state, it can differ strongly for different CVs. In general, most novae and the members of the UX UMa subtype of novalike variables reveal modest amplitudes of the order of 0.1 (in white light), while the VY Scl stars, i.e. those novalike systems which occasionally drop into a low state, appear to have systematically a more violent flickering of several tenths of a magnitude. The flickering in outbursting dwarf novae resembles that of UX UMa stars, while in quiescence they exhibit a large total range of amplitudes. In some cases it is so violent, that flares of more than a magnitude peak brightness rise and decay within just a few minutes.

Although being typical for CVs, flickering is not unique to cataclysmic variables. It is also observed in some symbiotic stars where a large part of the 


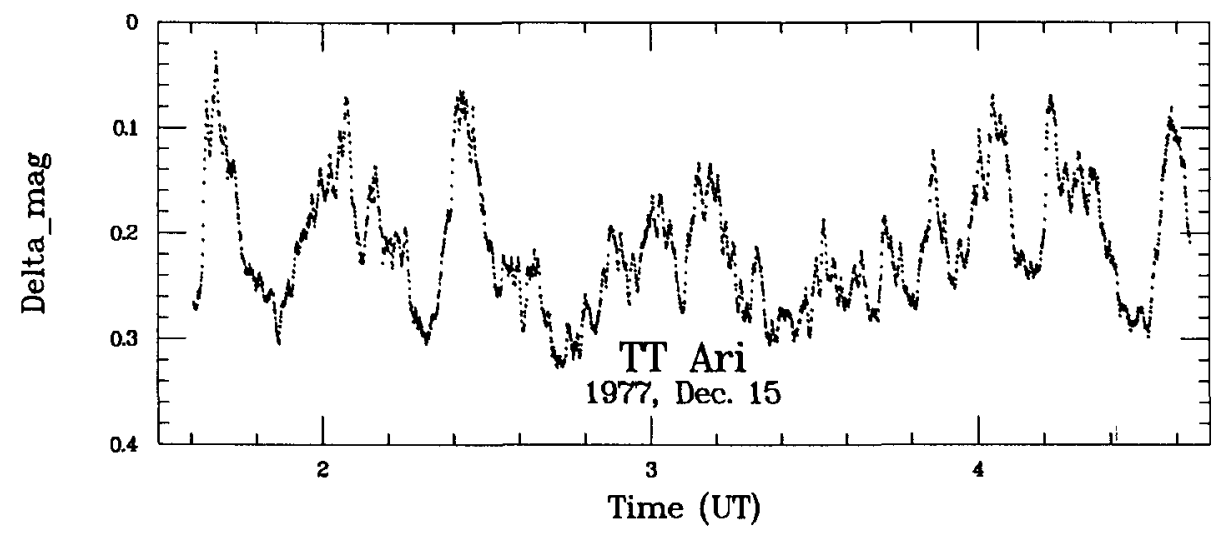

Fig. 1. Unfiltered differential light curve of the novalike variable TT Ari on 1977, Dec. 15.

luminosity is suspected to be powered by accretion. Other candidates showing this phenomenon are low-mass X-ray binaries where, however, with the exception of the case of Sco X-1 it is by far not as well documented as in CVs. Light curves of $\mathrm{T}$ Tau stars with accretion disks also show irregular variations (Gahm 1995) which resemble very much the flickering in CVs and can be regarded as its counterpart. It appears thus that the accretion process is ultimately responsible for flickering.

The often considerable amplitudes of the flickering indicate immediately that a significant fraction of the total visual light of a CV - sometimes half of it or even more - is emitted by the flickering light source. I therefore want to make a point saying that we cannot claim to understand the physics of a CV unless we understand what fickering is. In spite of this obvious fact, and although the phenomenon is known for more that 40 years, there are scarcely any systematic investigations of the flickering. Most of the corresponding statements in the literature (with very few exceptions) are restricted to a mere qualitative description or at most to the derivation of some rather superficial statistical parameters.

The first encompassing attempt to quantify flickering, to derive general properties and to find constraints for a physical model were performed by Bruch (1992). It was somewhat hampered by the fact that the observational material available to him was not really sufficient neither in quantity nor in quality. In order to improve the situation, no new observations are required, but one can draw upon the numerous light curves of CVs observed in the past which were never investigated with respect to the properties of the flickering. With the kind support of colleagues all over the world I could collect a data base of about 1400 light curves suitable for flickering studies, representing about 140 days and nights of continuous observing time. This material is currently being investigated, and the first results are just beginning to emerge. 


\section{The quantification of flickering}

One can choose different approaches to quantify flickering. On the one hand a flickering light curve can be regarded as an ensemble of data, of which one can obtain global characteristics. This approach involves the study of power spectra and the like. On the other hand we can try to identify individual flares in the light curves, perform statistics with them and deduce properties of the flickering in this way. Both approaches have their benefits.

The most serious difficulties opposed to a systematic study of flickering are problems which affect many physical measurements, but which are particularly annoying in the present connection:

- Noise: Just as noise, flickering is a stochastic process. Moreover, its amplitude is the smaller, the shorter the regarded time scales are. Therefore, flickering below a certain limit in time scale and amplitude level - which depends on the noise level in the data - is practically indistinguishable from noise. At the same time, just because noise and flickering cannot be distinguished, this limit is not easily quantifiable. This means also that flickering in different light curves with different noise levels is difficult to compare.

- Time resolution: Flickering occurs on time scales up to about $20^{\mathrm{m}}-30^{\mathrm{m}}$. A lower limit is unknown. The high frequency part of the flickering can only be studied if the time resolution of the measurements is adequately high. A sufficient time resolution is particularly important if individual flares are to be recognized. Again, it is obviously difficult to compare the flickering in light curves observed with different time resolutions.

In the general case the problems due to noise and time resolution get mixed, which certainly does not facilitate the quantitative analysis!

The problem of noise can in certain cases be solved by a Fourier analysis of the light curves. As an example, Fig. 2 shows a power spectrum of a light curve of KR Aur on a double logarithmic scale. It is typical for power spectra of flickering light curves. There is a linear drop from low to high frequencies, which reflects that larger events in the flickering occur on longer time scales. At a certain frequency, however, the power becomes constant. This is not due to flickering having no power at high frequencies but to white noise which prevails over flickering here. To estimate the noise level, one can compare this power spectrum to that of a simulated constant signal, subjected to Poisson noise. If the count rate of the constant signal is chosen such that the high frequency parts of both power spectra coincide, the corresponding Poisson noise is equal the noise in the real data.

But the power spectra of flickering light curves serve also other purposes. The slope of the decreasing part contains information about the flickering. Similarly, a wavelet analysis can tell us something about the properties of the flickering. Corresponding investigations are currently being carried out. It is, however, too early to report about results.

In another approach flickering can statistically be regarded as a Markov process. The individual data points in a light curve are transferred into symbolic 


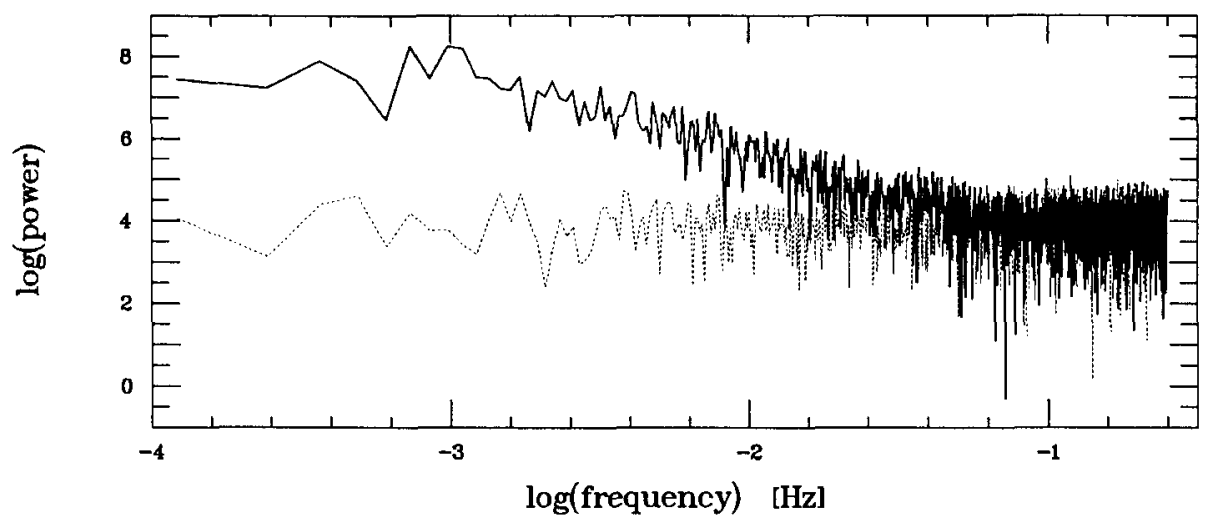

Fig. 2. Power spectrum of the light curve of KR Aur of 1977, Nov. 14 (solid line) and of an artificial constant signal, subjected to Poisson noise, chosen such that its high frequency part coincides with that of the real light curve (broken line).

observations which are then elements of a Markov chain. At any point in the light curve the system is considered to be in one of several states. Going to the next data point there is a certain (unknown) probability to remain in the same state or to make a transition to another state. This probability depends only on the state the system is currently in. In the first step, we investigate the simplest case of three states: (1) getting brighter, (2) remaining constant (within the noise limits), or (3) getting fainter.

Using the observations it is then possible to calculate a statistical model for the process driving flickering. Comparing such models for different light curves, one can define a measure for the distance of the models, i.e. for the similarity of the light curves. Light curves of similar statistical properties have a small, such with strongly differing characteristics a large distance. While we do not yet understand well how to interpret these differences physically, for the time being we regard them as an empirical measure for the similarity of different light curves.

Before statistics with individual flares in a light curve can be performed, it is necessary to devise a method to identify flares in the presence of noise and in view of the available time resolution. There is no unique way to do this, and therefore any method will be arbitrary to a certain degree. But this can be suffered as long as it is secured that this arbitrariness introduces only a minimum of systematic errors into light curves of different qualities. A prescription for flare identification has been given by Bruch (1992). To avoid problems with noise, it purposely disregards flares below a pre-defined amplitude limit.

The goal of all efforts to quantify flickering must be to gain a physical understanding of the underlying mechanism (or mechanisms?). A way to learn something about it is to look for correlations among the flickering properties and other system parameters. A most interesting result of this kind is a rela- 


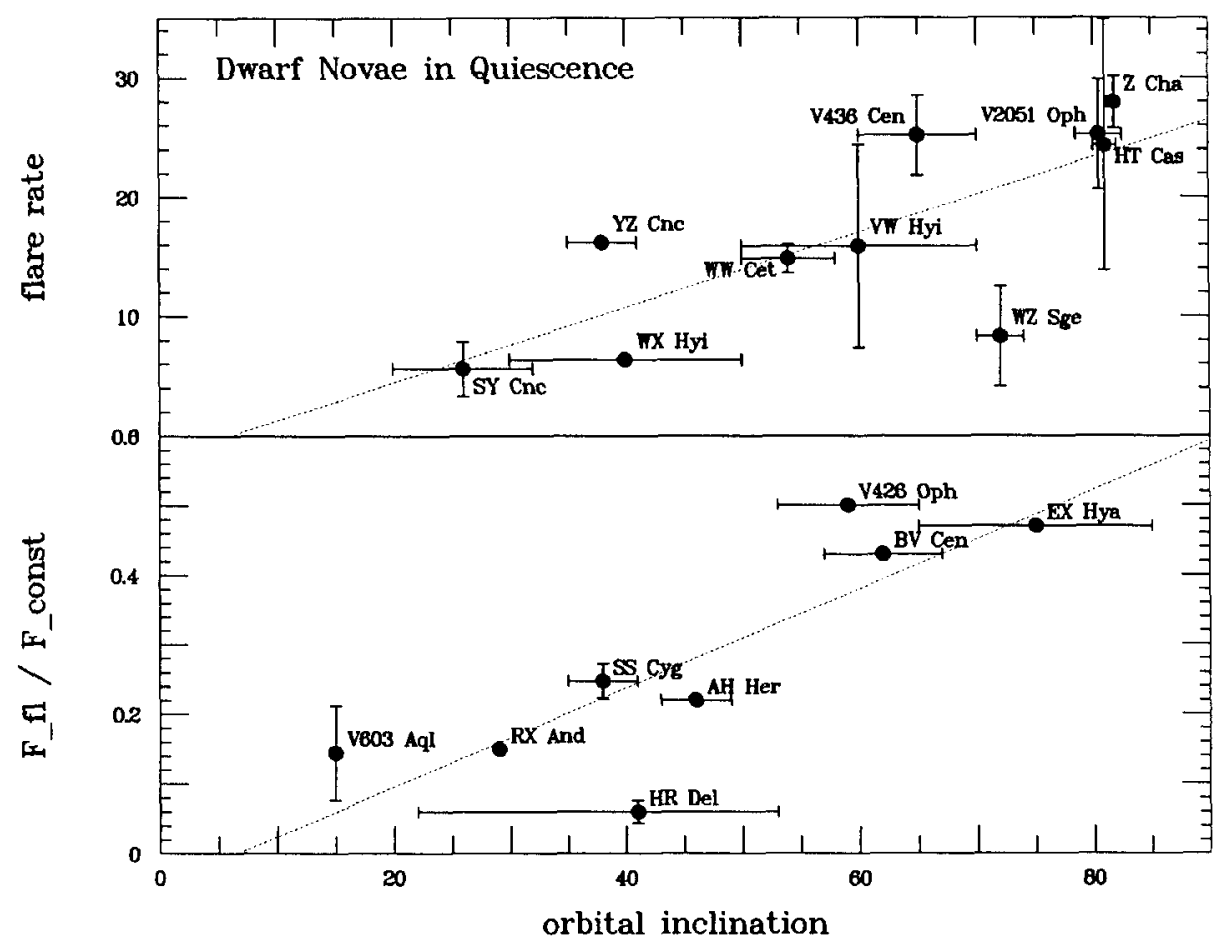

Fig. 3. The flare rate in quiescent dwarf novae (upper frame) and the ratio between the optical luminosity of the flickering light source and the constant part of the accretion disk (lower frame), both as a function of the orbital inclination.

tion between the flare rate - that is the number of individual flares per time unit - and the orbital inclination in quiescent dwarf novae (see Fig. 3; upper frame). The flare rate (measured in light curves given in magnitudes as opposed to fluxes) is large if we see the system at a high inclination. This supports a result of Bruch (1992) who found that the ratio between the optical luminosity of the flickering light source and the constant part of the accretion disk also increases with the inclination (Fig. 3; lower frame). These findings contain some important clues to the origin of flickering (see Sect. 3).

Another interesting point is the wavelength dependence of the flickering. It is normally described to be blue, but this statement is rarely quantified. Measuring the amplitudes of flares in different bands of a well-calibrated photometric system, it is possible to determine the broad band spectrum of the flickering light source. In Fig. 4 the spectrum derived from various light curves of VY Scl observed in the Walraven system is shown. The results for other CVs look similar. The solid lines indicate spectra of simple steady state accretion disk models, using the black body approximation and various mass transfer rates. All spectra are normalized to the $B$-band. The flickering spectrum rises steeper to the 


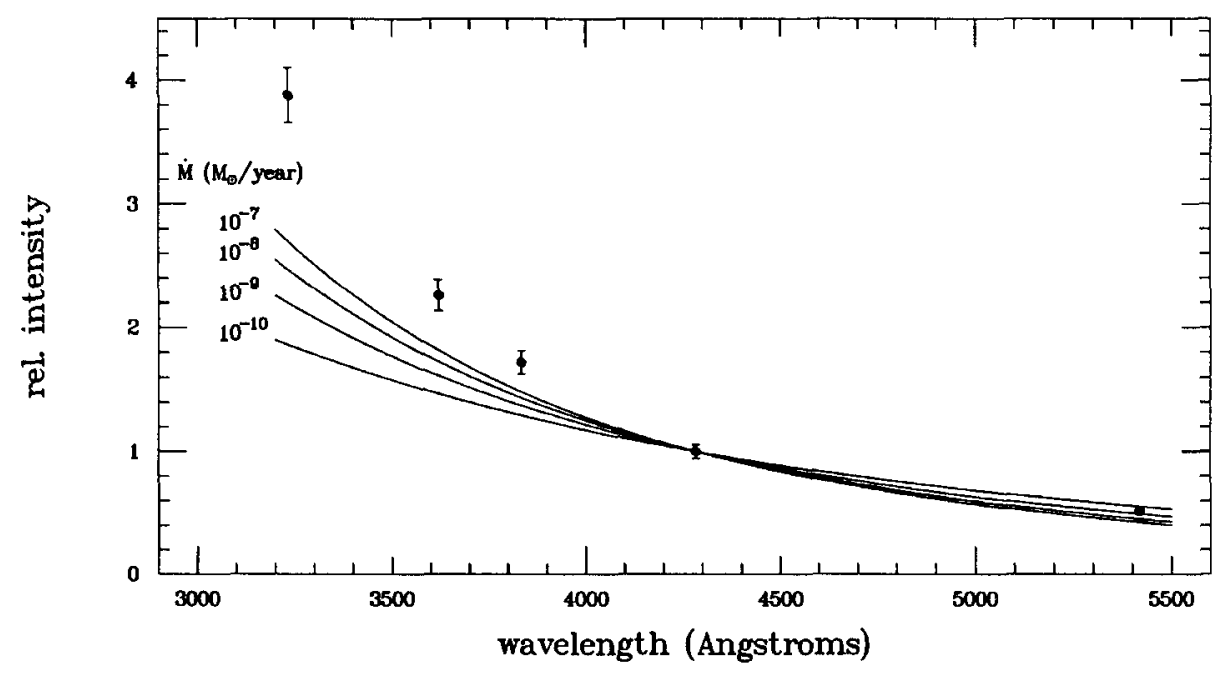

Fig. 4. Broad band spectrum of the flickering light source in the novalike variable VY Scl (dots) compared to steady state accretion disk spectra, assuming the black body approximation and different mass transfer rates (solid lines). All spectra are normalized to the mean wavelength of the $B$-band of the Walraven system.

UV than the simple disk model. This is also true for black bodies or power law spectra, regardless of the assumed temperatures or spectral indices, respectively. The flickering spectra suggest that the relative contribution of the corresponding light source to the total radiation is even stronger in the ultraviolet range than in the optical, enhancing the importance of the flickering light source for the total emitted radiation of a CV. UV photometry with a suitable time resolution is only available for the system AE Aqr (Eracleous et al. 1994). There, the amplitude of the flickering still increases a bit from the near UV to the middle UV, but not dramatically so. But then, AE Aqr is in many respects a very unusual system, and the behaviour of its flickering may not be representative for all CVs.

\section{The site of the flickering light source}

For some time, flickering has been explained as due to an unsteady transfer of matter from the secondary star, causing a variable luminosity of the hot spot. But there are many reasons why this cannot be the correct mechanism, at least not the principal one. Bruch (1992) has shown that the kinetic energy of the impact between the transferred matter and the accretion disk is not sufficient to explain the high fraction of the luminosity of the flickering light source on the total luminosity of the CVs. Moreover, the temperature of the hot spot is measured to be of the order of $10000 \ldots 15000 \mathrm{~K}$, less than the temperature of 
the inner accretion disk which dominates the blue light of a CV. It is then hard to see how the hot spot as the flickering light source could be even bluer than the steady accretion disk.

A direct indication that the flickering arises in other parts of the system than the hot spot is seen in the light curves of some eclipsing systems which permit to distinguish the ingress and the egress of the white dwarf and the hot spot, respectively. At phases when the hot spot is eclipsed by the secondary, but the white dwarf and the inner accretion disk are not, flickering is clearly seen. In other systems, the strength of the flickering, measured as the standard deviation from the mean curve, is strongly reduced at eclipse centre, indicating that the light source of the flickering is centred on the white dwarf but has a smaller extension than the accretion disk (Horne \& Stiening, 1985). Recently, Welsh \& Wood (1995) have performed eclipse mapping of the flickering light source in HT Cas and found that the flickering occurs in the inner accretion disk and peaks on the white dwarf.

The ultimate energy source for the flickering must be potential energy of the gravitational field of the white dwarf. In order to release the gravitational energy, matter in the accretion disk has to sink down which has to occur unsteadily in order to explain flickering.

Of course, most gravitational energy is available close to the white dwarf. Rough energy budget calculations performed by Bruch (1992) indicate that relatively large turbulent eddies in the inner accretion disk may have just enough energy to explain the luminosity of the flickering in the optical range. Also, the time scales on which the flickering flares occur are compatible with dynamical time scales in such turbulences. However, if a correction is made for the energy which is likely emitted in the UV range, it becomes difficult to explain flickering by turbulences in the disk alone. There are no problems with the energetics, however, if an unsteady and turbulent dumping of matter onto the surface of the white dwarf is assumed. Such a scenario appears to be the only one which is not at odds with the observed properties of the flickering, although a definite physical mechanism of how this unsteady accretion occurs is not yet known. It may well be possible that the interaction of the plasma in the inner accretion disk with a magnetic field of the white dwarf (which is not necessarily strong enough to cause directly observable effects as in polars or intermediate polars) is involved.

Obviously, the structure of the boundary layer between the (Keplerian) accretion disk and the white dwarf plays an important role in this connection. The boundary layer belongs to the least understood parts of CVs. There are some theoretical calculations concerning its structure. But they all make simplifying assumptions or neglect potentially important physical effects such as the influence of a magnetic field of the white dwarf. There are good arguments for a boundary layer not being restricted to a narrow equatorial belt, but engulfing the white dwarf to a considerable extent or even entirely (Livio \& Truran 1990). Unsteady accretion out of such a boundary layer could explain why the relative contribution of the flickering light source and also the flare rate increase 
with increasing orbital inclination: While at high inclinations the disk is seen foreshortened and thus less luminous than at low inclinations, this is not the case for the flickering light source. Thus, a larger number of flares will have an amplitude (on the magnitude scale) above a given level, and the ratio between flickering and constant light increases.

If the boundary layer would in fact be very thin, it should have a very high temperature and emit mostly in X-rays. Observations indicate that both, the temperature and the $\mathrm{X}$-ray emission are significantly lower than predicted (for references, see Bruch \& Duschl 1993). Duschl \& Tscharnuter (1991) therefore assumed a radially extended boundary layer and investigated the consequences of this scenario. Persuing this idea further, Bruch \& Duschl (1993) calculated the luminosity ratio between the Keplerian part of the accretion disk and the boundary layer as a function of the boundary layer radius. Assuming the flickering to be in fact due to a turbulent accretion of matter onto the white dwarf and thus defining a lower limit for the boundary layer luminosity, it is then possible to estimate a lower limit for the radius of the extended boundary layer by comparing the flickering luminosity with that of the quiet disk which is interpreted as the Keplerian part. In this way the radial extent of the boundary layer was found to be at least of the order of 2 or 3 white dwarf radii or even a bit more. This result compares favourably with the radius of the flickering light source determined by eclipse tomography in HT Cas (Welsh \& Wood 1995).

We still cannot claim to have gained a satisfactory understanding of flickering in cataclysmic variables. But doubtlessly some progress in deriving a systematic picture of the empirical properties has been achieved in recent times, and certainly we will still learn quite a lot in this respect. A scenario for the flickering is beginning to emerge. Hopefully, the quantification of the observed characteristics will finally lead to a consistent physical model.

Acknowledgements: It is a pleasure to express my thanks to my collaborators S. Beckemper, T. Fritz, J. Kinscher, G. Lichtenberg, M. Niehues, C. Nohlen, M. Sand and T. Schimpke who study the flickering together with me, and to the numerous colleagues worldwide who put their light curves of cataclysmic variables at my disposal.

\section{References}

Bruch A., 1992, A\&A 266,237

Bruch A., Duschl W.J., 1993, A\&A 275, 219

Duschl W.J., Tscharnuter W.M., 1991, A\&A 241, 153

Eracleous M., Horne K., Robinson E.L., Zhang E.-H., Marsh T.R., Wood J.H., 1994, ApJ 433, 313

Gahm G., 1995, this volume p. 203

Horne K., Stiening R.F., 1985, MNRAS 216, 933

Livio M., Truran J.W., 1990, Comm. Astrophys. 14, 221

Welsh W.F., Wood J.H., 1995, this volume p. 300 